\title{
Auditory Decisions in the Supplementary Motor Area
}

Isaac Morán, ${ }^{1 \S}$ Javier Perez-Orive, ${ }^{2 \S}$ Jonathan Melchor, ${ }^{1}$ Tonatiuh Figueroa, ${ }^{1}$ and Luis Lemus ${ }^{1} *$

${ }^{1}$ Department of Cognitive Neuroscience, Institute of Cell Physiology, Universidad Nacional Autónoma de México (UNAM). 04510. Mexico City, Mexico.

${ }^{2}$ Instituto Nacional de Rehabilitacion "Luis Guillermo Ibarra Ibarra”. Mexico City, Mexico.

$\S$ These authors contributed equally

* Corresponding author

lemus@ifc.unam.mx

Telephone: (+52) 5556225675

\section{Keywords}

Word recognition, choices, premotor cortex, macaque, psychophysics 


\section{Abstract}

In human speech and communication across various species, recognizing and categorizing sounds is fundamental for the selection of appropriate behaviors. But how does the brain decide which action to perform based on sounds? We explored whether the premotor supplementary motor area (SMA), responsible for linking sensory information to motor programs, also accounts for auditory-driven decision making. To this end, we trained two rhesus monkeys to discriminate

6 between numerous naturalistic sounds and words learned as target (T) or non-target (nT) categories. We demonstrated that the neural population is organized differently during the auditory and the movement periods of the task, implying that it is performing different

9 computations in each period. We found that SMA neurons perform acoustic-decision-related 10 computations that transition from auditory to movement representations in this task. Our results 11 suggest that the SMA integrates sensory information while listening to auditory stimuli in order 12 to form categorical signals that drive behavior.

\section{Introduction}

16 Recognition and categorization of sensory information are essential for deciding which action to

17 take (Parker \& Newsome, 1998; Freedman et al., 2001; Romo et al., 2003; Seger \& Miller,

18 2010). In particular, categorizing dynamic sounds into classes is necessary for social

19 communication in various species (Prather et al., 2009; Miller et al., 2003), including monkeys

20 (May, Moody \& Stebbins, 1989) and humans, where grouping of discrete sounds into words is

21 fundamental for speech recognition (Repp, 1984; Leonard \& Chang, 2014). However, it is not

22 clear how the brain links the recognition of complex sounds to behavior. In primates, auditory

23 information is carried from the auditory cortex to the prefrontal and premotor cortices via ventral

24 and dorsal auditory streams (Romanski et al., 1999; Kusmierek \& Rauschecker, 2014). Recent

25 studies with macaques have shown that premotor regions may represent an embodiment of

26 acoustic recognitions (Archakov et al., 2020). The premotor supplementary motor area (SMA)

27 has been linked to voluntary action and cognitive control of movement (Nachev et al., 2008;

28 Lara et al., 2018; Shima \& Tanji, 2000). The SMA also participates in working memory and

29 decision making during tactile discrimination (Romo et al., 1997; Hernandez et al., 2002; Lemus 
et al., 2007), imagery, and it links auditory information with motor programs (Lima et al., 2016; Vergara et al., 2016). Similarly, in songbirds, the premotor HVC nucleus is involved in coding complex sounds and orchestrates singing (Prather et al., 2009).

Nevertheless, few studies have been published on naturalistic sound coding (Rauschecker, 1998; Gentner \& Margoliash, 2003; Tsunada et al., 2011; Town et al., 2018), and even fewer in behaving non-human primates (Ng et al., 2009; Scott et al., 2012; Chandrasekaran et al., 2013; Melchor et al., 2020). This could be because there is still plenty to learn from experiments using simple, non-naturalistic sounds that do not require behavior (e.g. pure tones, amplitude-modulated noise; Liang et al., 2002; Selezneva et al., 2006; Bendor \& Wang, 2008; Yin et al., 2020). Additionally, although macaques have brain organizations that are similar to those of humans and although they, too, use vocalizations to communicate (Chang et al., 2010; Daube et al., 2019), some consider that non-human primates are challenging for training and studying auditory behavior (Ng et al., 2009; Scott et al., 2012). However, Melchor et al. (2020) recently showed that macaques attend to and memorize numerous naturalistic sounds. Therefore, in macaques, the SMA is amenable for studying the neural correlates of decisions within an auditory categorization task.

Here we present data from extracellular recordings of SMA neurons from two rhesus monkeys trained to discriminate between two groups, or categories, of naturalistic sounds and then choose an action. We also tested acoustic morphings to reveal the underlying responses to linear physical changes rather than discrete categorizations. We found robust categorical responses at both the single neuron and population levels. A small number of neurons were also found to carry information about individual sensory stimuli during the auditory period. By mapping the neural population activity during different periods of the task, we found that it organizes into orthogonal subspaces during the task's auditory and movement periods. Finally, the linear morphings of one stimulus into another indicate that the coding in SMA changes from sensory, during the auditory period, to categorical during the movement period. Taken together, our results support the hypothesis that SMA integrates auditory information necessary for a categorization that provides action signals for behavioral choices. 


\section{Results}

Monkeys discriminate between learned naturalistic sounds

We trained two monkeys (Macaca mulatta) to perform an auditory recognition task. During the task, the monkeys obtained a drop of liquid as a reward for releasing the lever only after identifying a target sound (T). A T followed 0,1 , or 2 non-target sounds (nT). In other words, the T could appear in three different temporal positions (P1, P2, and P3; Figs. 1a,c, see Methods). It is important to note that we incorporated a delay and a green visual cue (VC) after each sound to disentangle auditory and movement signals. Thus, after a $\mathrm{T}$, the monkeys waited for a $0.5 \mathrm{~s}$ delay before a $0.5 \mathrm{~s}$ VC-release period (Figs. 1a,c). The monkeys learned, by trial and error, to recognize up to fourteen sounds (half of them T, half $\mathrm{nT}$ ) belonging to four different classes: conspecific vocalizations, interspecific vocalizations, words, and artificial sounds (Figs. 1b,d; see Methods). Once the monkeys performed above $85 \%$ correct responses (Fig. 1d), we recorded single neurons (1341 from monkey 1; 524 from monkey 2) from SMA (Fig. 1c (inset) during task execution).

Single units and population activity represent naturalistic sounds

Rasters of a characteristic SMA neuron (Fig. 2a) show a substantial firing rate change during a $\mathrm{T}$ regardless of its position (i.e. P1, P2, or P3). In other words, the responses show a clear distinction between $\mathrm{T}$ and $\mathrm{nT}$, but with small or no differences within each category (Figs. $2 \mathrm{~b}$ and c). The majority of neurons recorded in both monkeys (68.5\% and $85.9 \%$, respectively) exhibited this type of categorical coding (Fig.2d), as measured with the f-statistic and bootstrapping (see Methods). Interestingly, a small percentage of neurons displayed significant coding for specific sensory stimuli within the $\mathrm{T}$ and $\mathrm{nT}$ categories $(2.4 \%$ and $1.1 \%$ for $\mathrm{T} ; 1.1 \%$ and $2.1 \%$ for nT in each monkey, respectively; Fig. 2d). As shown in Figures 2b, 2c, and 2e, neurons with this particular sensory coding did this during the auditory periods when sounds were presented $(0.0-0.5 \mathrm{~s})$. In contrast, the f-statistic shows that the categorical coding starting at

90 the end of auditory periods rises further during the delays (0.5-1.0 s) and movement periods

$91 \quad(1.0-1.5$ s; Fig. 2e). 
93 To study the neuronal population dynamics, we used principal component analysis (PCA), a

94 dimensionality reduction technique. Similarly to the observed results of individual neurons, the

95 population dynamics differentiated between $\mathrm{T}$ and $\mathrm{nT}$, independently of the position in which $\mathrm{T}$

96 appears (Fig. 3a). The component explaining most of the variance shows a small increase at the

97 beginning of the T stimulus, followed by a larger increase during the movement period, as

98 expected for this premotor area. The second component shows acoustic-anticipatory activity that

99 continues to rise with Ts but decreases for nTs, providing a clear auditory-based signal for

100 premotor activity. The third principal component shows a large increase at the beginning of the

101 trial and an additional increase before the lever release indicating the end of the trial.

102

We also used demixed PCA (dPCA), a dimensionality reduction tool that can separate the

104 effects on the population response of different task parameters (Kobak et al., 2016), to separate

105 the effect of the auditory stimulus coding from the condition-independent activity (Fig.3b). Since

106 the previous results from single units showed strong categorical codification, we computed

107 dPCA for the T and nT categories. The demixed principal components (dPCs) with the most

108 weight explaining the variance of the population activity are those for the categorical coding

109 (first two categorical coding dPCs explain $57.7 \%$ and $65.3 \%$ of the total variance for each

110 monkey, respectively, while the first two condition-independent dPCs explain $29.6 \%$ and $29.5 \%$

111 of the variance). For both monkeys, the dPCs separated the T and nT trajectories during the

112 auditory periods. For $\mathrm{dPC} 1$, this separation persisted during the delay and reached its maximum

113 during the movement period (Fig.3b, top). Here, dPC2 and dPC3 shift their signs in the delay or

114 during the movement period. The neural activity projections over the condition-independent

$115 \mathrm{dPCs}$ are associated mainly with the task's temporal dynamics.

117 Auditory and movement computations occupy different subspaces

119 After observing the differences between auditory and movement period responses of single

120 neurons and the SMA population, we set out to determine whether such representations

121 proceeded from distinct population organizations, each coming together at different time periods

122 to perform different computations (Elsayed et al., 2016). Figure 3c highlights the correlation 
123 matrices for the full neural population, organized so the structure during the auditory period is

124 highlighted, while keeping this same ordering for the movement period. The correlation matrices

125 show two large groups of neurons for both monkeys, with high correlations during the auditory

126 period, but not during the movement period (Fig.3c). To evaluate whether this difference was

127 due to two different populations, we calculated a simple epoch-preference index (Elsayed et al.,

128 2016; see Methods). If the preference index distribution for all neurons is bimodal, this implies

129 two pools of neurons. However, we found evidence that both monkeys' data came from a

130 unimodal distribution (Hartigan's dip test $\mathrm{p}=0.98$ and $\mathrm{p}=0.96$ for monkeys 1 and 2 ,

131 respectively), suggesting that the auditory and movement activities did not come from two

132 different populations.

We used PCA to investigate whether these population responses during the auditory and movement epochs occupy partially overlapping or orthogonal subspaces within the neural

136 activity space. By definition, the top five PCs during the auditory period capture a large amount

137 of variance from this period. However, we found that they captured minimal variance from the

138 movement period (monkey 1: 76.9\% vs. 3.3\%; monkey 2: 88.8\% vs. $10.9 \%$, auditory vs.

139 movement period variances, respectively, for PC1 to 5 combined; Fig.3d). Similarly, the PCs

140 calculated for the movement period captured a large proportion of movement period variance,

141 but a minimum of auditory period variance (monkey 1: 4.8\% vs. 86.5\%; monkey 2: $12.0 \%$ vs.

$14292.8 \%$, auditory- vs. movement-period variances, respectively, for PC1 to 5; Fig.3d). This result

143 supports the hypothesis that the auditory and movement periods use close to orthogonal

144 subspaces to perform their computations.

We also calculated an alignment index to quantify the subspace overlap between the

147 activities of both signals. Here, an index of zero means no alignment and one full alignment. The

148 alignment index was 0.08 and 0.23 for monkeys 1 and 2, respectively, significantly lower than an

149 alignment index calculated in a random neural sampling distribution ( $\mathrm{p}<0.05$ for both

150 monkeys). This result indicates that the population rearranges its activity for auditory and

151 movement periods in a nearly orthogonal manner. Therefore, since the neuronal population's

152 activity behaves in an almost orthogonal fashion in the auditory and movement periods, it is

153 possible to implement a linear decoder to generate two orthogonal subspaces in order to explain 
154 the neuronal variability population in both periods. Thus, we applied an algorithm (Elsayed et al.,

155 2016) to find two sets of bases that identify principal components of each period in an orthogonal

156 manner (Figs. 3e and f). The top four PCs from the auditory subspace captured $63.7 \%$ and $75.8 \%$

157 of the auditory variance in monkeys 1 and 2, respectively. The top four PCs from the movement

158 subspace captured $76.7 \%$ and $82.0 \%$ of the movement variance for monkeys 1 and 2,

159 respectively. Plotting one against the other revealed that the $\mathrm{T}$ and $\mathrm{nT}$ categories separate and

160 move in opposite directions during the auditory period. Meanwhile, T elicits a broad trajectory

161 during the movement period while nT remains close to the origin (Figs. $3 \mathrm{~g}$ and $\mathrm{h}$ ). There is a

162 striking similarity between the monkeys' results, indicating that the appearance of these different

163 population organizations at different times in the task is not monkey-dependent.

Linear stimulus changes modulate behavior and neural activity

167 Gradually morphing sounds into one another (Fig.4a) may reveal the degree to which the 168 neuronal activity changes as a function of the physical dimensions of the stimuli or reflect the 169 behavioral outcome computations. By fitting sigmoidal functions to the monkeys' behavior (Fig.

$1704 \mathrm{~b}$; Table 1) and the neuronal responses to the morphings (Figs. 4c-d) it is possible to observe the 171 relationship between the neuronal activity and behavior. The neuronal responses of an example 172 neuron (Fig. 3c) identified T at morphing values between 60\% and 100\%, and nT at morphing 173 values between $0 \%$ and $40 \%$. Remarkably, a stimulus at $50 \%$ produced a neuronal response 174 between the two groups. However, there was a gradual change during the auditory period, 175 whereas the responses transition more abruptly in the movement period. Figure $4 \mathrm{~d}$ shows this 176 more precisely. The neurometric and psychometric functions are compared (gray and black lines, 177 respectively) during the auditory, delay, and movement periods. During the latter periods of the 178 task, the neurometric curves of all recorded neurons became more similar to the psychometric 179 curve, i.e. with steeper slopes around the 50\% midpoint (Fig.4e). The overall ratios of the 180 neurometric/psychometric midpoint slopes increased progressively from the auditory, to the 181 delay, to the movement periods: $0.47,0.60$, and 0.93 for monkey 1 , and $0.38,0.69$, and 0.92 for 182 monkey 2. Furthermore, figure 4f shows increasing Spearman correlations between neurometric 183 and psychometric curves for all recorded neurons as the task progresses. These results imply that 184 SMA neurons have more information about sensory stimuli during the auditory period and more 
185

213 To study whether the SMA codes for acoustic decisions, we trained two monkeys to categorize 214 naturalistic sounds. Observing the time courses of the neural responses, analyzed in different

215 ways, we present here several lines of evidence supporting the concept that SMA integrates

categorical information (thus becoming more similar to the monkeys' behavior) during the movement period.

Demixed PCA revealed that, in response to morphing stimuli, the population activity followed a pattern similar to that of the single neurons, distinguishing between $\mathrm{T}$ and $\mathrm{nT}$ and with an in-between response at 50\% morphing (Fig.4g). Divergence started during the auditory period, followed by a separation to two distinct attractors during the movement period (Figs. $4 \mathrm{~g}$ h). The two-dimensional dPC projections of the population activity (Fig.4H) show how neuronal responses to $\mathrm{T}$ and $\mathrm{nT}$ sounds form two groups and move towards one of two attractors. The transition from one attractor to the other occurred abruptly, further evidencing the SMA's role in the categorical coding of naturalistic sounds.

\section{The non-alert condition and errors also point to a role played by SMA in acoustic decisions}

To further distinguish between sensory and categorical coding, we projected the neural activity during the errors over the categorical dPCs calculated in correct responses. We found an inversion of activity resembling that of the correct trials (Fig. 5a). That is, in error trials the population activity in SMA reflected the behavioral decision made, rather than the sensory stimulus presented. Moreover, such population activity suggests that when a signal separation during the auditory period does not occur, monkeys make errors. We also recorded the neural activity during sets of passive listening, i.e. when the monkeys listened but did not perform the task because we withdrew the lever and the visual go-cues. Figure $5 \mathrm{~b}$ shows that during these sets, SMA ceased coding for auditory or categorical decisions. This result indicates that SMA is only active when the monkeys engage in the task, further supporting the role of SMA involvement during acoustic-driven decision making.

\section{Discussion}


216 sensory information during the auditory period, in support of categorical representations during

217 the movement period: A) Demixed PCA, in which the main dPC began to separate in the

218 auditory period, remained constant during the delay, and then rose sharply during the movement

219 period. B) The two different organizational states were indicative of two different computations

220 being performed in each, during the auditory and movement periods. C) Single neuron

221 recordings during morphing sets showed coding-shifts from sensory to categorical

222 representations as the task progressed. D) The activity of the neuronal population diverged to

223 two distinct attractors, i.e. T and nT. E) Trials in which the monkeys made errors suggested that

224 a separation in the neural population activity seems to be needed during the auditory period;

225 additionally during the movement period the neuronal population with false alarms, had the same

226 activity as during hits.

In previous work with monkeys trained to solve a delayed match-to-sample acoustic task,

229 Scott et al. (2012) reported that the monkeys performed poorly in such tasks. In our study,

230 although the monkeys' training was more prolonged than in visual or tactile paradigms (Lemus

231 et al., 2009), the monkeys ended up being highly proficient in the categorization of numerous

232 naturalistic sounds (see also Melchor et al., 2020). Therefore, our task presents multiple

233 advantages for studying the neural basis of listening. However, even when the task comprised a

234 great diversity of complex sounds, the monkeys learned to include them in just two categories,

235 i.e. T and nT. Therefore, we did not explore each sound's semantics, but probably only

236 synonyms, i.e. that multiple sounds mean T, while others mean nT. Moreover, since the monkeys

237 expressed their decisions by releasing a lever or holding it down waiting for a $\mathrm{T}$, one could argue

238 that the monkeys only had to recognize one of the two categories of sounds. Nevertheless, this

239 argument is not plausible since SMA neurons, like the monkeys, acknowledged both categories.

As expected for a premotor area (Nachev et al., 2008; Lara et al., 2018; Shima \& Tanji,

242 2000), during the task's movement period, single neurons and population responses coded for the

243 monkeys' dichotomous decisions in the task. Furthermore, dPCA showed that the task's

244 categorical component captured most of the variance, as opposed to other commonly observed

245 results with this method, in which the condition-independent components are often those with the

246 most weight (Romo et al., 1999; Kobak et al., 2016). Interestingly, the second principal 
component (Fig. 3a) rises before each auditory stimulus in a predictable fashion, apparently consistent with models of accumulation of information (Leon \& Shadlen, 1999). Despite the fact that such a phenomenon, similar to a hazard function of the probability of appearance of a T, was not previously described for auditory processing in SMA, it is consistent with its role in premotor processing (Shima \& Tanji, 2000; Nachev et al., 2008; Zimnik et al., 2019). Furthermore, this is the first description of how SMA neuronal activity correlates with decisions based on active listening to naturalistic sounds.

Interestingly, a small group of neurons coded for sounds during auditory periods, as predicted by imaging studies that suggested that the premotor cortex is activated by auditory stimuli (Lima et al., 2016; Vergara et al., 2016; Archakov et al., 2020). Nevertheless, to our knowledge, our results constitute the first electrophysiological evidence of SMA coding natural sounds. Previous fMRI studies using acoustic morphings (Jiang et al., 2018) suggested a twostep category-learning process. First, a perceptual stage in the auditory cortex, and later a categorization stage in the lateral prefrontal cortex. While our current data do not contradict those findings, it adds further nuance by providing evidence that there is a separate computation that processes the physical features of sensory stimuli in premotor cortex. Elsayed et al. (2016) showed that a population of neurons in motor cortex acts as two coordinated circuits of preparatory and movement computations. When we applied their analytical method, we, too, found that the activity of a single neural population in SMA occupies orthogonal subspaces

267 during the auditory and movement periods, implying different computations in each period.

268 Therefore, those two periods' computations require no more than a single population of neurons organizing itself into two different circuits.

We presented the monkeys with sounds that morphed gradually into one another, in order

272 to determine how SMA responds to linearly changing physical stimuli. Measuring the slope at 273 the midpoint of the sigmoidal curve of the neurometric and psychometric curves, we found that 274 during the auditory period, the neurometric curves have smaller slopes than the psychometric 275 curve, implying that at this moment of the task the neural response is more gradual, and varies 276 more linearly with the changing sensory stimulus. During the movement period, both the neurometric and the psychometric curves had similar slopes, indicating that the neural responses 
are as categorical as the behavioral choices the animals make. From a population perspective, dPCA shows that sounds are grouped into two clear categories, with the $\mathrm{dPC}$ of the $50 \%$ response in between these groups. Furthermore, as revealed by the first and second principal components, the population response evolves towards two distinct attractors corresponding to the $\mathrm{T}$ and $\mathrm{nT}$ categories. The transition from one of these attractors to the other is abrupt rather than gradual, which is a hallmark of categorical coding (Wills et al., 2005; Freedman et al., 2001).

Taken together, our results show how the SMA participates in naturalistic sound-driven decision making by integrating acoustic information to produce decision signals and motor commands. Future work in other cortical areas that lie upstream from the SMA may provide additional information with regard to how this categorization occurs.

\section{Methods}

\section{Ethics statement}

293 Animals were handled following the Official Mexican Standard for the Care and Use of

294 Laboratory Animals (NOM-062-ZOO-1999) and the study was approved by the Internal

295 Committee for the Use and Care of Laboratory Animals of the Institute of Cell Physiology,

296 UNAM (CICUAL; LLS80-16).

298 Animals and experimental setup

299 Two adult rhesus macaques (Macaca mulatta; monkey 1: a 10-year-old male weighing $13 \mathrm{~kg}$;

300 Monkey 2: a 10-year-old female weighing $7 \mathrm{~kg}$ ) participated in the experiments. Training and

301 recording sessions took place in a soundproof booth. Each monkey sat on a primate chair with its

302 head fixed at a distance of $60 \mathrm{~cm}$ from a 21 color LCD monitor $(1920 \mathrm{x} 1080$ resolution, $60 \mathrm{~Hz}$

303 refresh rate). A Yamaha MSP5 speaker (0.05-40 kHz frequency range) was situated fifteen

304 centimeters above and behind the monitor delivering sounds at $\sim 65 \mathrm{~dB}$ SPL (measured at the

305 monkeys' ear level). Additionally, a Logitech ${ }^{\circledR}$ Z120 speaker was placed directly below the

306 Yamaha speaker to generate background white noise at $55 \mathrm{~dB}$ SPL. Finally, a metal lever

307 placed at the monkeys' waists captured their responses. 
310 We trained two rhesus macaques to perform an acoustic recognition task. During the task, the

311 monkeys categorized sounds as either Target (T) or non-target (nT). In a trial, a gray circle of $3^{\circ}$

312 aperture appeared at the center of the screen. Then, a sequence of one to three sounds arose. Each

$3130.5 \mathrm{~s}$ sound continued with a $0.5 \mathrm{~s}$ delay and then a $0.5 \mathrm{~s}$ green visual cue that replaced the initial

314 gray circle. The cue indicated that the monkey should release the lever if the sound consisted of a

$315 \mathrm{~T}$. The monkeys received a reward for releasing the lever in a time window of $0.7 \mathrm{~s}$ from the start

316 of the T's cue. However, after an nT, the monkey kept the lever pressed, waiting for a T. If the

317 monkey released the lever before a $\mathrm{T}$, the trial was classified as a false alarm, and a new one

318 commenced. We required that the monkeys' performance in this task remain above an $80 \%$ hit

319 rate before the electrophysiological recordings were made. The programming task was done in

320 LabVIEW 2014 (64-bit SP1, National Instruments®).

\section{Auditory stimuli}

323 The sounds were recorded in our laboratory or downloaded from open access online libraries

324 (https://freesound.org/). The monkeys learned more than 37 sounds from 4 different categories

325 (Melchor el al., 2020): conspecific vocalizations, heterospecific vocalizations, words, and

326 artificial sounds. However, for statistical repetitions the recordings were made with only 14

327 sounds $(\mathrm{T}=7 ; \mathrm{nT}=7)$ : conspecific vocalizations $(\mathrm{T}=2 ; \mathrm{nT}=1)$, interspecific vocalizations $(\mathrm{T}=$

$3281 ; \mathrm{nT}=1)$ and words $(\mathrm{T}=2 ; \mathrm{nT}=3)$. The sounds' sample rate was $44.1 \mathrm{kHz}$; their amplitudes

329 were normalized at -10dB SPL (RMS), and their durations were shortened or lengthened to $0.5 \mathrm{~s}$

330 (Adobe Audition®).

\section{Morphing sounds}

333 We created sets of morphing sounds that change from an $\mathrm{nT}$ to a $\mathrm{T}$ in a continuum sequence

334 (STRAIGHT software; Kawahara et al., 1999). This procedure consists of a linear interpolation

335 of five spectro-temporal characteristics of a pair of sounds, i.e. fundamental frequencies,

336 formants, duration, spectro-temporal density, and aperiodicity. The resulting sounds contained

337 some percentage of stimulus A and a complementary percentage of B. Thus, each set consisted

338 of seven morphed sounds (i.e., 02040506080 and $100 \%$ T). The monkeys trained in various

339 sets; however, monkeys 1 and 2 executed 6 and 4 sets during recordings. 
Passive condition

343 In this condition, the sounds appeared in a continuous clip separated by $0.3 \mathrm{~s}$ interlapses, and

344 with no other cue or visual stimulus. The monkeys did not perform since the lever was not

345 present. However, the monkeys received a reward randomly every 15-30 s.

\section{Neural recordings}

348 Extracellular recordings of neurons proceeded from an array of five independently movable 349 microelectrodes (1-3 M $\Omega$; Thomas Recording $($ ) inserted at different SMA locations in each

350 session. We positioned $20 \mathrm{~mm}$ diameter recording chambers according to the Paxinos brain atlas

351 coordinates (Paxinos et al., 2000) and structural magnetic resonance imaging of both monkeys

352 (interaural $27 \mathrm{~mm}$, left hemisphere: lateral $6 \mathrm{~mm}$ ). The sampled extracellular membrane

353 potentials were $40 \mathrm{kHz}$ (Plexon®). Isolation of individual neurons was performed manually

354 using Plexon offline sorter software (Plexon®; monkey 1) and automatically using MountainSort

355 (Chung et al., 2017; monkey 2).

357 Data analysis

358 Individual neural coding

359 To evaluate whether SMA neurons coded for T and nT categories or the identity of particular 360 sounds, we performed F-statistics in a one-way ANOVA of the T vs. nT dependent activity and

361 individual sounds. We aligned the neuronal activity to the start of sounds and calculated the 362 mean firing rate in 200-ms windows sliding in steps of $20 \mathrm{~ms}$. To avoid biases in the F-statistic 363 due to comparing classes of different numbers of trials, we equalized the number of trials using 364 the lowest common number of trials in all classes. We repeated the analysis 1000 times to create 365 an F-statistic distribution. To determine the time bins where the F-statistic was significant, we 366 compared the actual distribution against an F-statistic random distribution obtained by shuffling 367 the stimulus labels 1000 times. A neuron was significant if the $95 \%$ confidence intervals of the 368 actual and random distributions did not overlap in at least one time bin. 
We used principal component analysis (PCA) to describe the SMA's neuronal population dynamics during the task. We calculated the neurons' firing rate in blocks of correct trials of 1, 2, or 3 stimuli. We calculated the firing rate using a window of $200 \mathrm{~ms}$ moving in steps of $20 \mathrm{~ms}$.

374 Subsequently, we concatenated the firing rate of these three types of trials to obtain a matrix $M \in$ $375 \mathbb{R}^{N \times E T}$ of $\mathrm{N}$ number of neurons, E types of trials (with 1, 2, or 3 stimuli), and T total time points. Next, we used the demeaned firing rate of matrix M to calculate the principal components (PCs), considering each row as a variable.

Population analysis with demixed PCA

We performed demixed principal component analysis (dPCA; Kobak et al., 2016) in order to observe the effects of the task parameters on the population responses. During the supervised part, dPCA decomposes the neural activity for each variable using the covariance matrices of different marginalizations. The unsupervised analysis of dPCA was similar to that of the PCA, for each marginalization matrix. We marginalized the population activity for $\mathrm{T}$ and $\mathrm{nT}$ and time. We studied the neuronal activity within times from the starts of sounds to the monkeys' response, in $200 \mathrm{~ms}$ windows sliding every $20 \mathrm{~ms}$. The dPCA algorithm separates the matrices from the decoder (D) and the encoder (F) for each parameter of the task by minimizing the loss function:

$$
L_{\phi}=\left\|\underline{X}_{\phi}-F_{\phi} D_{\phi} \underline{X}\right\|
$$

Where $\phi$ denotes the marginalization for each parameter and $\underline{X}$ is the mean-centered 392 population matrix (the average of each neuron's activity is 0 ). Each component can be ordered

393 based on the amount of variance explained. The axes obtained by the decoder and encoder allow 394 reduction of the data in a few components that capture the most significant variance of each 395 parameter of the task.

We implemented a method to determine the time bins with statistically correct decoding 398 (Kobak et al., 2016). First, we separated the data into training and testing sets. We constructed 399 the testing set by randomly separating one trial from each category ( $\mathrm{T}$ and $\mathrm{nT}$ ). In contrast, the 400 training set was the average of the rest of the trials in each category. We performed dPCA from 401 the training set to obtain the decoding axes. Then we projected the testing set on these axes and 
402 classified them according to the closest category mean ( $\mathrm{T}$ or $\mathrm{nT}$ ). We repeated this procedure

4031000 times and measured the proportion of correct classification. We used 100 shuffles to

404 calculate the classification accuracies expected by chance. The shuffled distribution was

405 computed by choosing random trials between categories for each neuron. We ran the same

406 method described above and calculated the proportion of correct classifications for each

407 iteration. Then we compared the proportion of correct classifications between the real data vs.

408 the shuffled data. The time bins where the real classification exceeded the shuffled classification

409 distribution were considered statistically different from chance.

Identifying different subspaces in auditory and movement periods

412 To compare the population states during the auditory and movement periods, we performed four

413 procedures to describe the transition between one to other state and a new algorithm developed

414 by Elsayed et al. (2016) to calculate optimally two orthogonal subspaces, where each one

415 captures auditory and movement activity. 1) To compare the population covariation between

416 auditory and movement periods, we computed the correlation between all neurons in each

417 period. We created two matrices for each period: $A \in R^{N \times C T}$ and $M \in R^{N \times C T}$ where $\mathrm{N}$ is the

418 number of neurons, $\mathrm{C}$ is the number of stimuli and $\mathrm{T}$ is the time. The correlation matrix of each

419 period was obtained computing the Pearson correlation between the rows of each matrix (A and

420 M). 2) To quantify the subspace overlapping between subspaces of both periods, we computed a

421 PCA over $A \in R^{N \times C T}$ and $M \in R^{N \times C T}$ to obtain the top ten auditory and movement PCs. First,

422 the firing rate of each period was projected onto the auditory PCs and the percentage of

423 explained variance relative to the total variance of the auditory period was quantified. Then, the

424 same procedure was executed for movement PCs. 3) We calculated an alignment index to

425 measure the amount of auditory period variance captured by the top ten movement PCs

426 normalized by the auditory period variance captured by the top ten auditory PCs. The index

427 range varied from 0 to 1 , where 0 indicates the orthogonality between both periods and 1 means

428 a perfect alignment. 4) To rule out that two groups of neurons are activated differentially in the

429 auditory and movement periods we use an epoch-preference index, which evaluates the relative

430 weight of each neuron in both periods. We calculated the firing rate range separately in each

431 period and divided it by the mean of the firing rate of the entire trial. Then the range of each

432 neuron was normalized by the mean of the firing rate of all neurons. A neuron with a positive 
433 value indicates a preference for the auditory period over the movement period, a negative value

434 indicates the opposite. A bimodal distribution of the index signifies the existence of two neural

435 subpopulations, one that is more selective for auditory period and other for movement period.

436 Finally, we used a method developed by Elsayed et al. (2016) to derive two orthogonal

437 subspaces, each one capturing auditory and movement activity, respectively. This method

438 maximizes the sum of the variance of each period activity in each period subspace and was

439 calculated with the following loss function:

$$
\left[\hat{Q}_{a u d}, \hat{Q}_{m o v}\right]=\operatorname{argmax}_{\left[Q_{a u d}, Q_{m o v}\right]} \frac{1}{2}\left(\frac{\operatorname{Tr}\left(Q_{a u d}^{T} C_{a u d} Q_{a u d}\right)}{\sum_{i=1}^{d_{a u d}} \sigma_{a u d}(i)}+\frac{\operatorname{Tr}\left(Q_{m o v}^{T} C_{m o v} Q_{m o v}\right)}{\sum_{i=1}^{d_{\text {mov }}} \sigma_{\text {mov }}(i)}\right)
$$

Where $C_{a u d}$ and $C_{m o v}$ are covariance matrices of the neural activity in auditory and

444 movement period, respectively. $\sigma_{a u d}(i) \mathrm{y} \sigma_{m o v}(i)$ are ith singular value of the $C_{a u d}$ and $C_{m o v}$,

445 respectively. $Q_{a u d} \mathrm{y} Q_{m o v}$ are the eigenvectors of auditory and movement subspaces. We

446 selected four dimensions for $Q_{a u d}$ and $Q_{m o v}$ due they capture close to $70 \%$ variance of each

447 period. This method identifies simultaneously the auditory and movement subspaces while

448 constraining them to be orthogonal.

\section{Psychometric and Neurometric Measures}

451 We adjusted hyperbolic tangent functions to the psychophysical and neurometric performance

452 during morphing sets. This function relates the percentage of times in which the monkey

453 (psychometric curve) or the neuron (neurometric curve) recognized a sound like a $\mathrm{T}$ as a function 454 of a morphing sound. The function is:

$$
f\left(\%_{\text {morph }}\right)=\beta 0+\beta 2 * \tanh \left(\beta 1 *\left(\%_{\text {morph }}-\theta\right)\right)
$$

$456 \theta$ and $\beta 0$ represent the midpoint of the function on the horizontal and vertical axis, respectively,

$457 \quad \beta 2$ represents the amplitude function from $\beta 0$, and $\beta 1$ defines the morphing percentage dependent 458 of behavior, a high value indicates an acute sigmoid. Additionally, we measured a linear slope at 459 the sigmoid's midpoint to assess the degree of perceptual and neural modulation as a function of 460 the morphing rate. We calculated the maximum and minimum values of the sigmoid's second 461 derivative to find the central part of the sigmoid. This section corresponds to the behavioral 
transition in the categorization of morphing stimuli. After obtaining the abscissa's and the ordinate's coordinates, we used the following equation to determine the slope:

$$
\text { slope }=\frac{y_{2}-y_{1}}{x_{2}-x_{1}}
$$

Neurometric function

468 We derived the neurometric functions from each neuron's correct trials (de Lafuente \& Romo,

469 2005). A neurometric curve represents the probability that an ideal observer reports the stimulus

470 category ( $\mathrm{T}$ or $\mathrm{nT}$ ) based solely on the firing rate. For each neuron and time bin, the neurometric

471 curve was the percentage of trials in which the maximum firing rate reached or exceeded an

472 arbitrary threshold. The threshold was selected to maximize the number of correct answers. For

473 the neurometric curves, we used $300 \mathrm{~ms}$ windows, sliding every $50 \mathrm{~ms}$. Psychometric and

474 neurometric sensitivity were compared based on sigmoidal slopes. We used a Spearman's

475 correlation between the raw data from the psychometric and neurometric curves.

\section{Acknowledgements}

478 We thank Elizabeth Cabrera for valuable comments. We are also grateful for the financial 479 support provided by CONACYT, CB-256767, and Programa de Apoyo a Proyectos de 480 Investigación e Innovación Tecnológica (PAPIIT) IN207919. Special thanks to Gerardo Coello 481 and Ana Escalante of the computing department of the IFC, to Gabriel Pérez Ruelas for technical 482 assistance, and to Mr Patrick Weill for proofreading the manuscript. Isaac Morán Martinez is a 483 doctoral student from Programa de Doctorado en Ciencias Biomédicas, Universidad Nacional 484 Autónoma de México (UNAM) and received fellowship 288348 from CONACYT, México. Data 485 in this work are part of his doctoral dissertation.

\section{Author Contributions}


Disclosure

493 The authors declare no conflicts of interest.

\section{References}

Archakov, D., DeWitt, I., Kuśmierek, P., Ortiz-Rios, M., Cameron, D., Cui, D., ... Rauschecker, J. P.

Bendor, D., \& Wang, X. (2008). Neural response properties of primary, rostral, and

Chung, J. E., Magland, J. F., Barnett, A. H., Tolosa, V. M., Tooker, A. C., Lee, K. Y., ... Greengard, L. F. (2017). A Fully Automated Approach to Spike Sorting. Neuron, 95(6), 1381-1394.e6. https://doi.org/10.1016/j.neuron.2017.08.030

De Lafuente, V., \& Romo, R. (2005). Neuronal correlates of subjective sensory experience. Nature Neuroscience, 8(12), 1698-1703. https://doi.org/10.1038/nn1587

Elsayed, G. F., Lara, A. H., Kaufman, M. T., Churchland, M. M., \& Cunningham, J. P. (2016). Reorganization between preparatory and movement population responses in motor cortex. Nature Communications, 7(1), 1-15. https://doi.org/10.1038/ncomms13239

Freedman, D. J., Riesenhuber, M., Poggio, T., \& Miller, E. K. (2001). Categorical representation 
of visual stimuli in the primate prefrontal cortex. Science, 291(5502), 312-316. https://doi.org/10.1126/science.291.5502.312

Gentner, T. Q., \& Margoliash, D. (2003). Neuronal populations and single cells representing learned auditory objects. Nature, 424(6949), 669-674. https://doi.org/10.1038/nature01731

Hernández, Adrián and Zainos, Antonio and Romo, R. (2002). Temporal Evolution of a DecisionMaking Process in Medial Premotor Cortex. Neuron, 33(6), 959-972. Retrieved from http://www.ncbi.nlm.nih.gov/pubmed/11906701

Jiang, X., Chevillet, M. A., Rauschecker, J. P., \& Riesenhuber, M. (2018). Training Humans to

Kawahara, H., Masuda-Katsuse, I., \& De Cheveigné, A. (1999). Restructuring speech

Kobak, D., Brendel, W., Constantinidis, C., Feierstein, C. E., Kepecs, A., Mainen, Z. F., ... Machens, C. K. (2016). Demixed principal component analysis of neural population data.

Kuśmierek, P., \& Rauschecker, J. P. (2014). Selectivity for space and time in early areas of the auditory dorsal stream in the rhesus monkey. Journal of Neurophysiology, 111(8), 16711685. https://doi.org/10.1152/jn.00436.2013

Lemus, L., Hernández, A., Luna, R., Zainos, A., Nácher, V., \& Romo, R. (2007). Neural correlates of a postponed decision report. Proceedings of the National Academy of Sciences of the United States of America, 104(43), 17174-17179.

549 Lemus, L., Hernández, A., \& Romo, R. (2009). Neural codes for perceptual discrimination of 
acoustic flutter in the primate auditory cortex. Proceedings of the National Academy of

Sciences of the United States of America, 106(23), 9471-9476. https://doi.org/10.1073/pnas.0904066106

Leon, M.I., \& Shadlen, M.N. (1999). Effect of expected reward magnitude on the response of neurons in the dorsolateral prefrontal cortex of the macaque. Neuron, 24(2), 415-425.

Leonard, M. K., \& Chang, E. F. (2014). Dynamic speech representations in the human temporal lobe. Trends in Cognitive Sciences, 18(9), 472-479. https://doi.org/10.1016/j.tics.2014.05.001

Liang, L., Lu, T., \& Wang, X. (2002). Neural representations of sinusoidal amplitude and frequency modulations in the primary auditory cortex of awake primates. Journal of Neurophysiology, 87(5), 2237-2261. https://doi.org/10.1152/jn.2002.87.5.2237

Lima, C. F., Krishnan, S., \& Scott, S. K. (2016). Roles of Supplementary Motor Areas in Auditory Processing and Auditory Imagery. Trends in Neurosciences, 39(8), 527-542. https://doi.org/10.1016/j.tins.2016.06.003 Communication Sounds By Japanese Macaques. Macaca Fuscata. Journal of the Acoustical

Miller, E. K., Nieder, A., Freedman, D. J., \& Wallis, J. D. (2003). Neural correlates of categories Society of America, 85(2), 837-847. https://doi.org/10.1121/1.397555

Melchor, J., Morán, I., Figueroa, T. and, \& Lemus, L. (2019). Perceptual Invariance of Words and

Nachev, P., Kennard, C., \& Husain, M. (2008). Functional role of the supplementary and presupplementary motor areas. Nature Reviews Neuroscience, 9(11), 856-869. https://doi.org/10.1038/nrn2478 varies with sound type. Hearing Research, 256(1-2), 64-74. 
Niessing, J., \& Friedrich, R. W. (2010). Olfactory pattern classification by discrete neuronal network states. Nature, 465(7294), 47-52. https://doi.org/10.1038/nature08961

Parker, A. J., \& Newsome, W. T. (1998). Sense and the single neuron: Probing the physiology of perception. Annual Review of Neuroscience, 21, 227-277. https://doi.org/10.1146/annurev.neuro.21.1.227

Paxinos, G., Huang, X.-F., \& Toga, A. W. (2000). The rhesus monkey brain in stereotaxic coordinates.

Prather, J. F., Nowicki, S., Anderson, R. C., Peters, S., \& Mooney, R. (2009). Neural correlates of categorical perception in learned vocal communication. Nature Neuroscience, 12(2), 221228. https://doi.org/10.1038/nn.2246

Quian Quiroga, R., \& Panzeri, S. (2009). Extracting information from neuronal populations: Information theory and decoding approaches. Nature Reviews Neuroscience, 10(3), 173185. https://doi.org/10.1038/nrn2578

Rauschecker, J. P. (1998). Cortical processing of complex sounds. Current Opinion in Neurobiology, 8(4), 516-521. https://doi.org/10.1016/S0959-4388(98)80040-8

Repp, B. H. (1984). Categorical perception: Issues, methods, findings. Speech and Language, 10, 243-335. https://doi.org/10.1017/СВ09781107415324.004

Romanski, L. M., Tian, B., Fritz, J., Mishkin, M., Goldman-Rakic, P. S., \& Rauschecker, J. P. (1999).

Romo, R., Brody, C. D., Hernández, A., \& Lemus, L. (1999). Neuronal correlates of parametric working memory in the prefrontal cortex. Nature, 399(6735), 470-473.

602 Romo, R., Merchant, H., Zainos, A., \& Hernández, A. (1997). Categorical perception of somesthetic stimuli: Psychophysical measurements correlated with neuronal events in primate medial premotor cortex. Cerebral Cortex, 7(4), 317-326. https://doi.org/10.1093/cercor/7.4.317

Romo, R., \& Salinas, E. (2003). Cognitive neuroscience: Flutter Discrimination: Neural codes, perception, memory and decision making. Nature Reviews Neuroscience, 4(3), 203-218. 
https://doi.org/10.1038/nrn1058

609

610

611

612

613

614

615

616

617

618

619

620

621

622

623

624

625

626

627

628

629

630

631

632

633

634

635

636

Scott, B. H., Mishkin, M., \& Yin, P. (2012). Monkeys have a limited form of short-term memory in audition. Proceedings of the National Academy of Sciences of the United States of America, 109(30), 12237-12241. https://doi.org/10.1073/pnas.1209685109

Seger, C. A., \& Miller, E. K. (2010). Category learning in the brain. Annual Review of Neuroscience, 33, 203-219. https://doi.org/10.1146/annurev.neuro.051508.135546

Selezneva, E., Scheich, H., \& Brosch, M. (2006). Dual Time Scales for Categorical Decision Making in Auditory Cortex. Current Biology, 16(24), 2428-2433. https://doi.org/10.1016/j.cub.2006.10.027

Shima, K., \& Tanji, J. (2000). Neuronal activity in the supplementary and presupplementary motor areas for temporal organization of multiple movements. Journal of Neurophysiology, 84(4), 2148-2160. https://doi.org/10.1152/jn.2000.84.4.2148

Town, S. M., Wood, K. C., \& Bizley, J. K. (2018). Sound identity is represented robustly in auditory cortex during perceptual constancy. Nature Communications, 9(1). https://doi.org/10.1038/s41467-018-07237-3

Tsunada, J., Lee, J. H., \& Cohen, Y. E. (2011). Representation of speech categories in the primate auditory cortex. Journal of Neurophysiology, 105(6), 2634-2646. https://doi.org/10.1152/jn.00037.2011

Vergara, J., Rivera, N., Rossi-Pool, R., \& Romo, R. (2016). A Neural Parametric Code for Storing Information of More than One Sensory Modality in Working Memory. Neuron, 89(1), 5462. https://doi.org/10.1016/j.neuron.2015.11.026

Wills, T. J., Lever, C., Cacucci, F., Burgess, N., \& O’Keefe, J. (2005). Attractor dynamics in the hippocampal representation of the local environment. Science, 308(5723), 873-876. https://doi.org/10.1126/science.1108905

Yin, P., Strait, D. L., Radtke-Schuller, S., Fritz, J. B., \& Shamma, S. A. (2020). Dynamics and Hierarchical Encoding of Non-compact Acoustic Categories in Auditory and Frontal Cortex. Current Biology, 30(9), 1649-1663.e5. https://doi.org/10.1016/j.cub.2020.02.047

Zimnik, A. J., Lara, A. H., \& Churchland, M. M. (2019). Perturbation of macaque supplementary motor area produces context-independent changes in the probability of movement 
initiation. Journal of Neuroscience, 39(17), 3217-3233.

\section{Figure Legends}

Figure 1. Acoustic recognition task and behavior

643 a) Sequence of events in a trial: The monkey pressed the lever after a gray visual circle indicated

644 the beginning of a trial. Then, a playback of one to three sequential sounds commenced. Each

645 sound lasted $0.5 \mathrm{~s}$ followed by a $0.5 \mathrm{~s}$ delay and a $0.5 \mathrm{~s}$ green visual circle (go cue). The monkey

646 received a reward for releasing the lever within a $0.7 \mathrm{~s}$ window after the green cue following a

647 Target sound (T) (red sonograms). False alarms occurred when the monkey released the lever

648 earlier, e.g. during a non-Target sound (nT) (blue sonograms). Finally, not releasing the lever

649 during the T response window counted as a miss trial. b) Examples of sonograms and

650 spectrograms of four $\mathrm{T}$ (top) and four nT (bottom) sounds. T and nT included four classes:

651 conspecific vocalizations, interspecific vocalizations, words, and artificial sounds. c) Types of

652 trials regarding the number of sounds. Inset, Brain scheme of MRI of monkey 2 showing the 653 location of the recording chamber over SMA. d) Percentage of correct responses for T (red) and

654 nT (blue) for each monkey (monkey 1: circles; monkey 2: triangles). The color code of stimuli 655 will be referenced in the following figures.

Figure 2. Individual SMA neurons code behavioral categories

658 a) Rasters and peristimulus time histograms (PSTHs) of a representative neuron from monkey 1.

659 Each row of ticks is one trial aligned with the lever press; each tick is an action potential. Each

660 plot corresponds to the possible number of sounds in a trial. Thick black ticks indicate the

661 beginning of a trial. Red ticks, lever pressings. b) Example PSTHs of two neurons for monkey 1

662 aligned with the start of the sounds. The color lines represent the PSTHs for each T and nT

663 stimulus. The lines below (black, categorical; red, T; blue, nT) indicate bins of significant

664 coding. T stimuli are depicted in red and nT in blue palettes, as in Figure 1d. The top neuron is

665 the same as in (a). c) Same as (b) but for monkey 2. d) Percentage of recorded SMA neurons

666 with statistically significant coding of behavioral categories (Cat) or sensory information (nT and

$667 \mathrm{~T}$ ), and for each monkey. e) Average F-statistic for selective SMA neurons as a function of time 
for both monkeys (monkeys 1: continuous lines; 2: dashed lines); Inset, a magnification showing small F-statistic values for the few neurons with significant sensory coding for particular T or nT sounds.

Figure 3. Categorical responses of SMA population project in auditory and movement orthogonal subspaces

674 a) Projections of the neural population responses in the first three principal components. The plot is grouped by the number of sounds, as in Figure 2a. Notice PC1 codes for lever release, PC2 for anticipation of sounds together with the likelihood of a T, and PC3 for Ts and movements. b) Left: First three dPCs for categorical coding. Each trace corresponds to T (red) and nT (blue) projections. Right: First three condition-independent dPCs. The numbers on the top right are the percentages of explained variance of all dPCs. The first categorical coding components explain a greater amount of variance compared to the condition-independent components. c) Correlation matrices for auditory (left) and movement (right) periods of all neurons of monkeys 1 (top) and 2 (bottom). Each dot corresponds to the correlation between two neurons. Matrices for both periods sorted as a function of the auditory period. The groups of correlated neurons that exist in the auditory period disappear in the movement period. d) Percentage of explained variance by each of the top five acoustic (left) and movement (right) PCs projected onto the population activity during auditory and movement periods. e) Projections of the neural population activity onto the first PC of the auditory subspace for monkeys 1 (top) and 2 (bottom). f) Same as (e), but for the movement subspace. g) Two-dimensional projection of the auditory and movement orthogonal subspaces for monkey 1. Circles and thick lines show the auditory and movement

690 periods, respectively. Arrows indicate flow of time. Same color code as in Figure 1d. h) Same as $691 \quad(\mathrm{~g})$, but for monkey 2.

Figure 4. Neural and behavioral responses to morphing sounds

694 a) Spectrogram examples of gradually morphing sounds from grunt (nT) to coo (T). Every morphing set comprises seven morphed sounds. The percentage of $\mathrm{T}$ contribution in each sound

696 is at the top of each spectrogram. b) Psychometric functions of monkeys (1: top; 2: bottom) as a 697 function of percentage of morphing. Each line is a sigmoidal fit to the behavioral performance. 698 The black line shows the mean performance for all morphings. c) Raster (top) and PSTHs 
699 (bottom) of a representative SMA neuron during the morphing set (grunt-coo) for monkey 2. d)

700 Psychometric (black) and neurometric (gray) comparison in three periods of the neuron in c:

701 auditory, delay, and movement. Each plot shows the percentage of times that behavior or

702 neuronal response considered each morph as T. e) Slope ratios (neurometric/psychometric) of all

703 neurons in three periods: auditory, delay, and movement. Monkeys 1: black, 2: gray. Dashed

704 lines and numbers represent means of the distributions (monkeys 1: black; 2: grey). Note that the

705 neurometric curves' slopes are smaller in the auditory period and become more similar to the

706 psychometric curves during the movement period. f) Time-dependent Spearman correlations

707 between neurometric and psychometric data points for both monkeys. g) Projection of the first

708 two stimulus dPCs in both monkeys (1: left; 2: right). Colors correspond to a particular

709 percentage of morphing (color code in c). h) Two-dimensional projection with the first two dPCs

710 of the population activity for both monkeys (1: left; 2: right) — color code as in g. Circles and

711 thick lines show auditory and movement periods, respectively. Arrows indicate time flow.

713 Figure 5. Error trials and the non-alert condition point to a participation of SMA in acoustic

714 decisions

715 a) The categorical dPCs calculated from correct trials (top) were used to project the population

716 neural activity in error trials (bottom) in monkey 1 . Note that when the dPCs do not separate

717 during the auditory period, the monkeys make errors. b) Projection of first dPC over the neural

718 population during passive trials shows no modulations.

720 Table 1. Overall fitting coefficients of psychometric curves. 
bioRxiv preprint doi: https://doi.org/10.1101/2020.10.20.347864; this version posted October 27, 2020. The copyright holder for this preprint (which was not certified by peer review) is the author/funder. All rights reserved. No reuse allowed without permission.

a

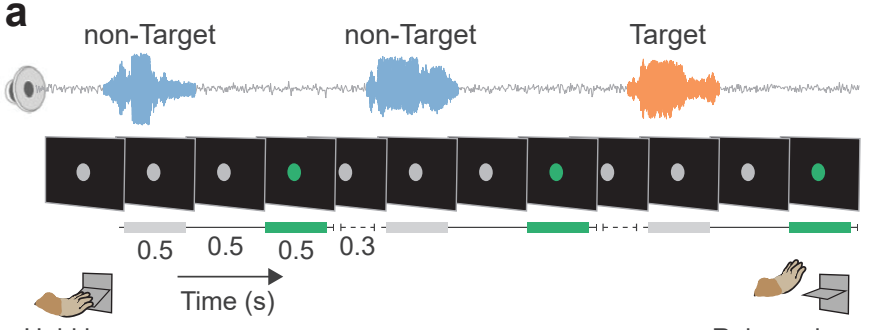

Hold lever

Release lever

b

coo warble moo [ko.'mi.ða]

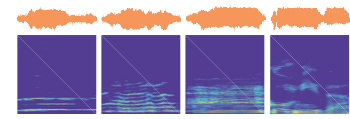

grunt woof [si] ['t]an.gi]

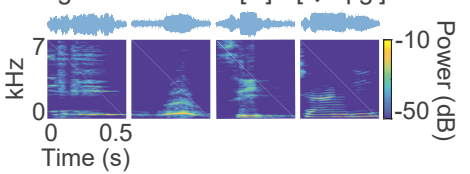

d

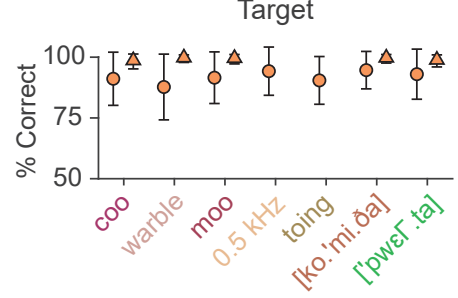

C

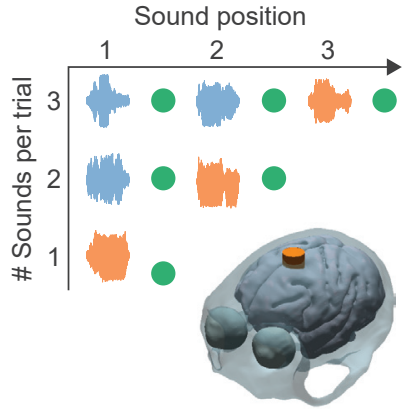

non-Target

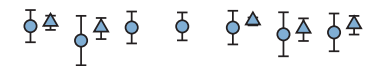

$\circ \because \triangle \because$

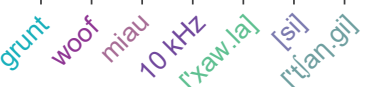

FIGURE 1 
bioRxiv preprint doi: https://doi.org/10.1101/2020.10.20.347864; this version posted October 27, 2020. The copyright holder for this preprint (which was not certified by peer review) is the author/funder. All rights reserved. No reuse allowed without permission.

a
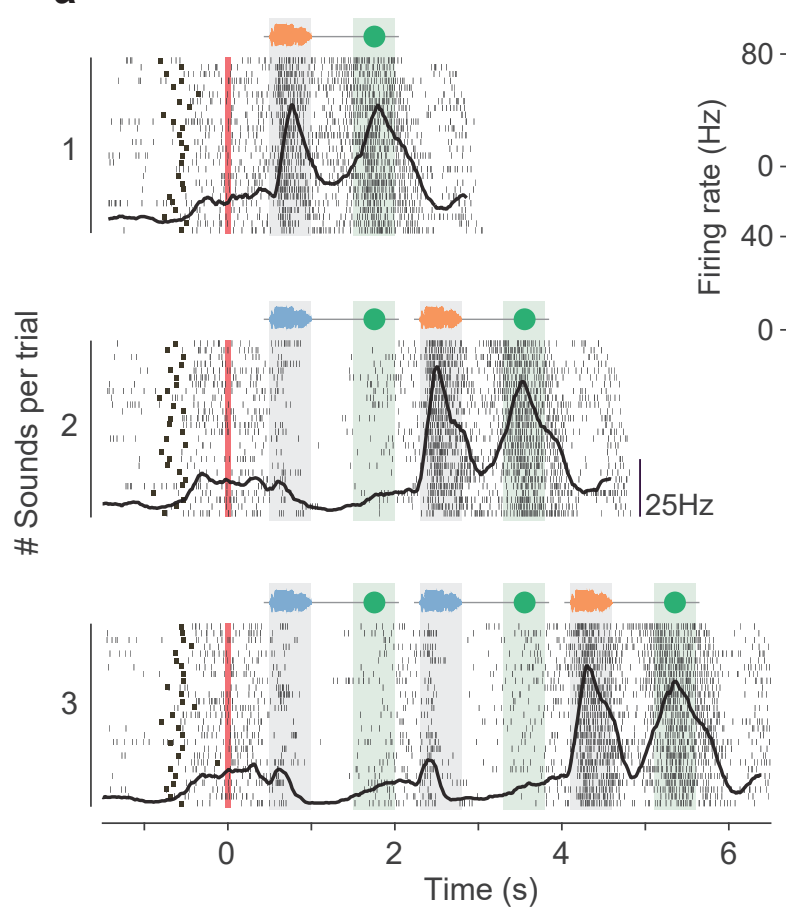

b

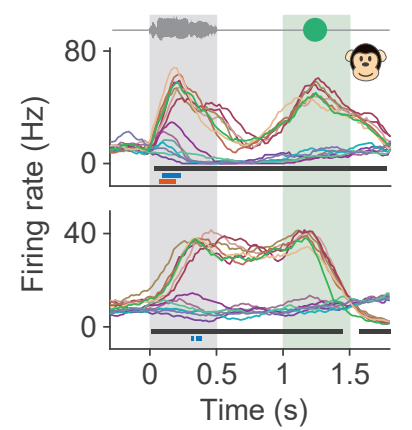

C



d

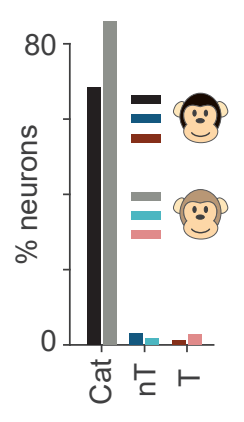

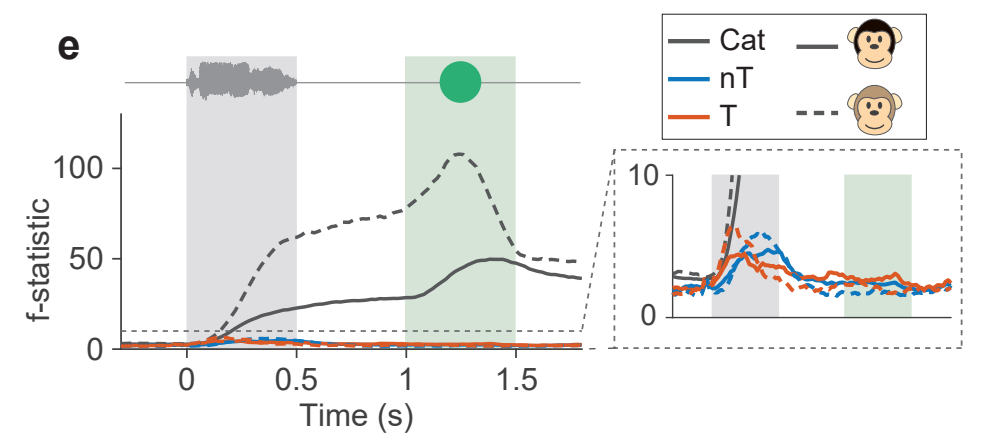

FIGURE 2 

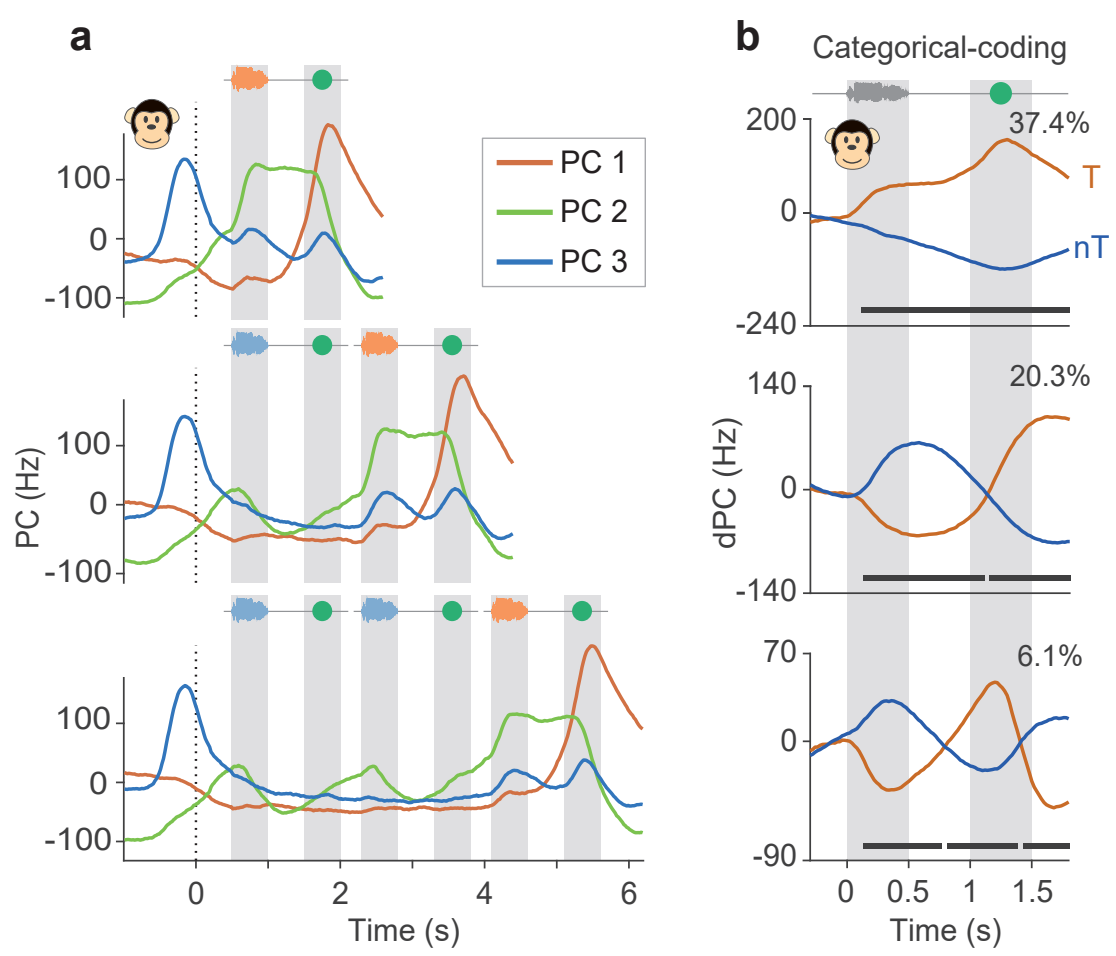

d

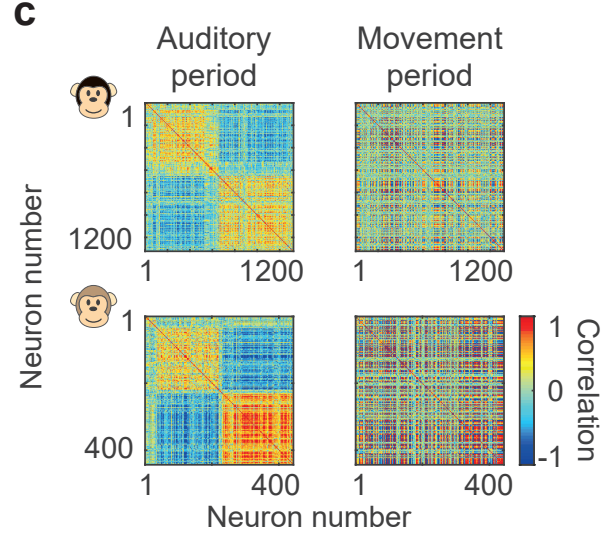

Condition-indepedent


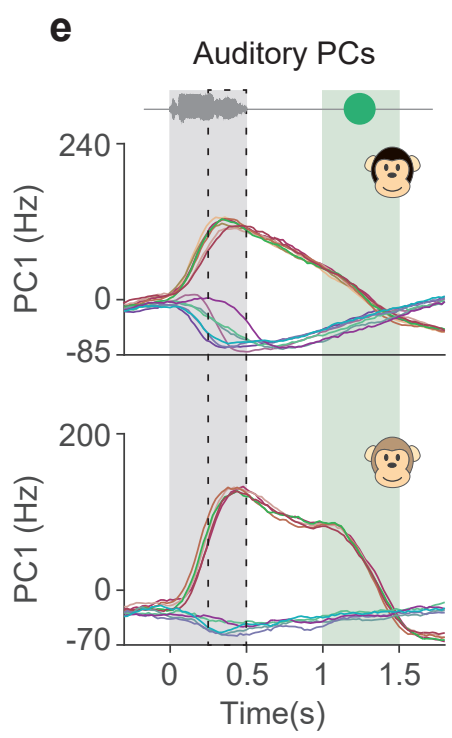

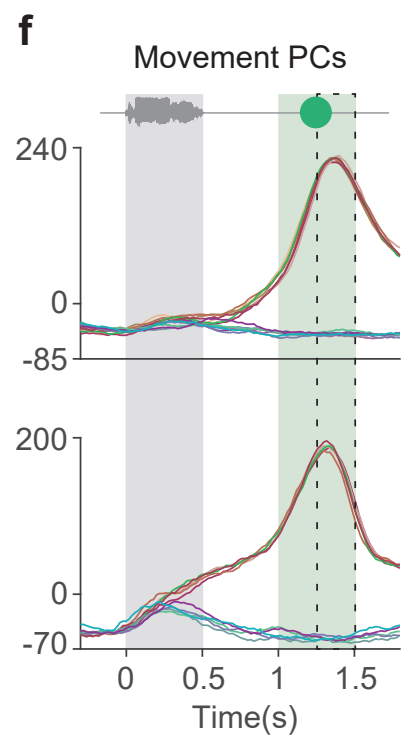
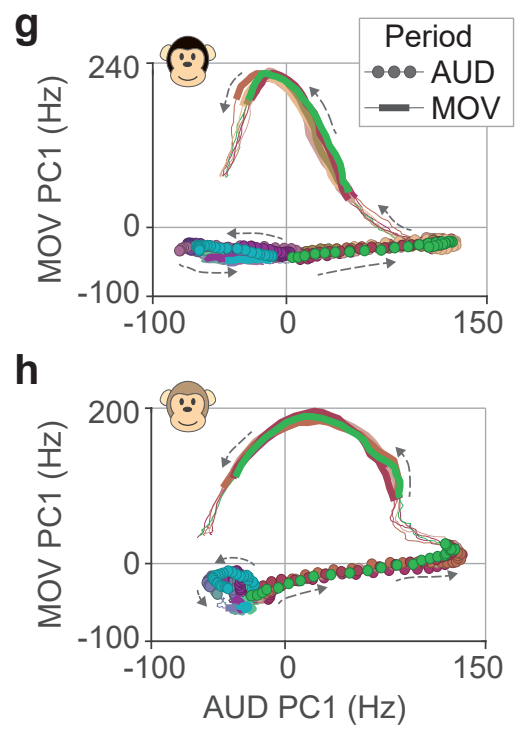

FIGURE 3 
bioRxiv preprint doi: https://doi.org/10.1101/2020.10.20.347864; this version posted October 27, 2020. The copyright holder for this preprint (which was not certified by peer review) is the author/funder. All rights reserved. No reuse allowed without permission.

a

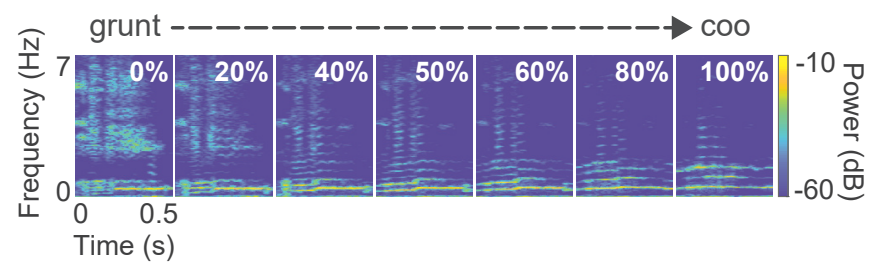

b

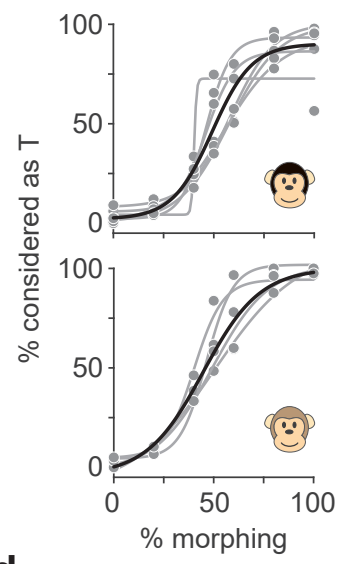

d

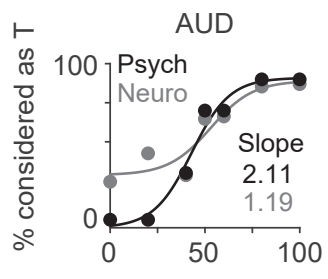

e

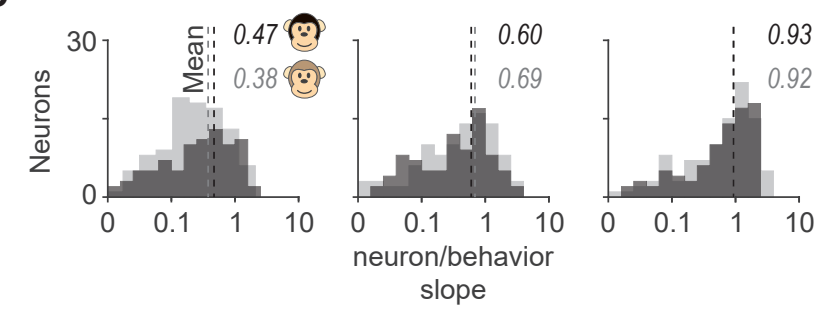

f

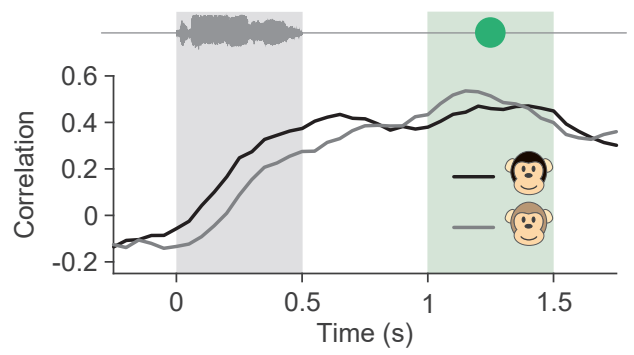

g
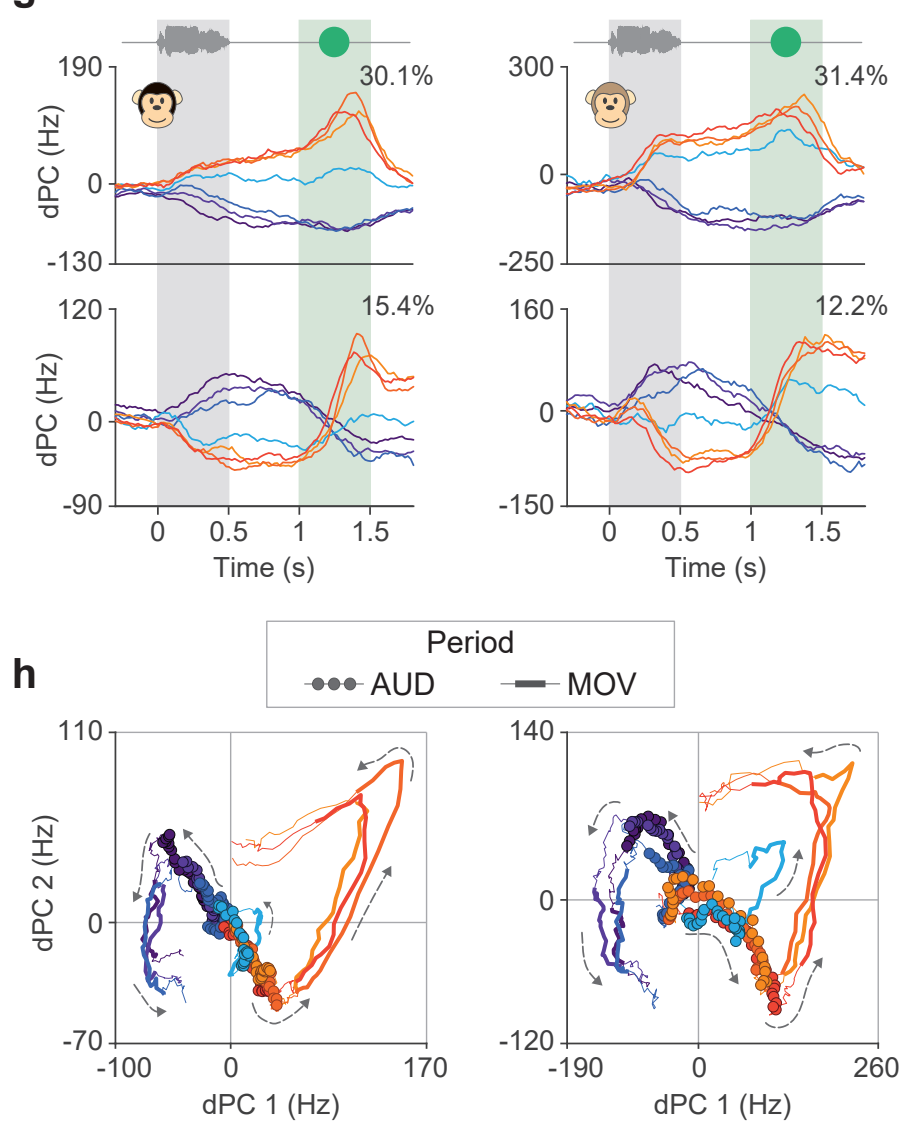

FIGURE 4 
bioRxiv preprint doi: https://doi.org/10.1101/2020.10.20.347864; this version posted October 27, 2020. The copyright holder for this preprint (which was not certified by peer review) is the author/funder. All rights reserved. No reuse allowed without permission.
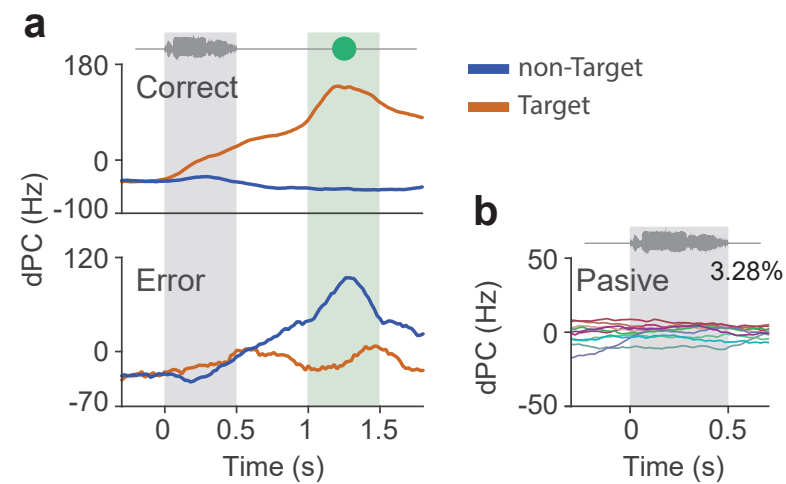

FIGURE 5 
bioRxiv preprint doi: https://doi.org/10.1101/2020.10.20.347864; this version posted October 27, 2020. The copyright holder for this preprint (which was not certified by peer review) is the author/funder. All rights reserved. No reuse allowed without permission.

\begin{tabular}{|c|c|c|c|c|c|c|c|c|c|c|}
\hline & non-Target & Target & $\begin{array}{c}\text { Number of } \\
\text { sessions }\end{array}$ & $\begin{array}{c}\text { Number of } \\
\text { neurons }\end{array}$ & Slope & $\theta$ & $\beta 0$ & $\beta 1$ & $\beta 2$ & $\mathrm{R}^{2}$ \\
\hline \multicolumn{11}{|l|}{ Monkey 1} \\
\hline & grunt & $\mathrm{coo}$ & 7 & 24 & 2.0 & 35.2 & 34.9 & 0.6 & 41.7 & 0.80 \\
\hline & grunt & moo & 6 & 36 & 1.9 & 60.3 & 51.3 & 0.5 & 51.0 & 0.91 \\
\hline & grunt & w arble & 12 & 57 & 2.3 & 45.6 & 47.3 & 0.5 & 51.7 & 0.96 \\
\hline & grunt & ['pw $\varepsilon \Gamma . t a]$ & 10 & 47 & 1.9 & 59.6 & 52.2 & 0.4 & 55.9 & 0.93 \\
\hline & grunt & [ko.'mi.ða] & 8 & 38 & 1.8 & 60.2 & 56.2 & 0.4 & 52.5 & 0.93 \\
\hline & [si] & moo & 2 & 10 & 1.6 & 46.7 & 46.6 & 0.4 & 45.5 & 0.94 \\
\hline \multicolumn{11}{|l|}{ Monkey 2} \\
\hline & grunt & $\mathrm{coO}$ & 13 & 91 & 1.6 & 52.9 & 52.0 & 0.3 & 59.4 & 0.96 \\
\hline & grunt & moo & 3 & 19 & 2.6 & 45.6 & 52.3 & 0.6 & 51.9 & 0.96 \\
\hline & grunt & w arble & 3 & 24 & 1.5 & 51.6 & 51.7 & 0.3 & 56.6 & 0.94 \\
\hline & ['xaw .la] & ['pw $\varepsilon \Gamma . t a]$ & 8 & 52 & 2.3 & 36.6 & 46.2 & 0.5 & 52.2 & 0.95 \\
\hline
\end{tabular}

Table 1 